\title{
Backtracking Search Optimization Algorithm for Synthesis of Concentric Circular Antenna Arrays
}

\author{
K. Guney, ${ }^{1}$ A. Durmus, ${ }^{2}$ and S. Basbug ${ }^{3}$ \\ ${ }^{1}$ Department of Electrical and Electronics Engineering, Faculty of Engineering, Nuh Naci Yazgan University, 38040 Kayseri, Turkey \\ ${ }^{2}$ Department of Electricity and Energy, Vocational College, Erciyes University, 38039 Kayseri, Turkey \\ ${ }^{3}$ Department of Computer Programming, Vocational College, Nevsehir University, 50300 Nevsehir, Turkey
}

Correspondence should be addressed to A. Durmus; alidurmus@gmail.com

Received 12 February 2014; Revised 17 June 2014; Accepted 8 August 2014; Published 4 September 2014

Academic Editor: Paolo Burghignoli

Copyright @ $2014 \mathrm{~K}$. Guney et al. This is an open access article distributed under the Creative Commons Attribution License, which permits unrestricted use, distribution, and reproduction in any medium, provided the original work is properly cited.

\begin{abstract}
A backtracking search optimization algorithm (BSA) is proposed for the synthesis of concentric circular antenna arrays (CCAAs) with the low sidelobe levels at a fixed beamwidth. Several numerical examples of CCAA patterns with the single, multiple, and broad nulls imposed at the directions of interference are also given to illustrate the performance and flexibility of the proposed algorithm. BSA is a relatively new population based evolutionary optimization algorithm. The numerical results show that the design of CCAA using BSA provides good sidelobe levels with a fixed beamwidth. The nulling patterns obtained by BSA are also very good. The quality of results obtained by the BSA is checked by comparing with that of several metaheuristic algorithms in the literature. BSA is also compared with differential search (DS) and bacterial foraging algorithms (BFA) in terms of iterative performances.
\end{abstract}

\section{Introduction}

It is well-known that the planar arrays have better control over the radiation pattern than the linear arrays. Besides other planar arrays such as circular and rectangular arrays, concentric circular antenna array (CCAA) has also become favourable in the radar and wireless communication systems. CCAA is capable of scanning electronically its main beam in the plane of the array without significant degradation of the array pattern. CCAA also has a good flexibility in terms of array pattern synthesis both in narrowband and in broadband applications [1-18].

For the antenna array synthesis problems, traditional optimization techniques involve particular disadvantages, owing to the nonlinearity. Starting points of optimization process become important to obtain good solutions if the dimension of the solution space is increased. They have also tendency to convergence to local optimum solutions. Additionally, gradient search methods also need continuous and differentiable cost functions [1]. For this reason, in recent years, the metaheuristic techniques $[1-3,19-34]$ based on the genetic algorithm, ant colony optimization, particle swarm optimization (PSO), differential evolution, tabu search, clonal selection, bees algorithm, harmony search, plant growth simulation, seeker optimization, evolutionary programming (EP), and bacteria foraging optimization (BFO) have become more popular, and they have been used in solving antenna array pattern synthesis problems. In this paper, an alternative metaheuristic technique based on a backtracking search optimization algorithm (BSA) [35] is employed for the synthesis of CCAA with the low sidelobe levels at a fixed beamwidth. Furthermore, several numerical examples of CCAA patterns with the single, multiple, and broad nulls imposed at the directions of interference are also presented. Additionally, in order to examine the computational performance of BSA, the convergence curve of BSA is compared with those of differential search (DS) [36] and bacterial foraging algorithm (BFA) [37].

The main motivation for the development of BSA is to achieve a simpler and more effective search algorithm [35]. Unlike many optimization algorithms, BSA has a single 
control parameter. Moreover, the problem solving performance of BSA is not oversensitive to the initial value of this parameter. BSA has a simple structure that is effective, fast, and capable of solving multimodal problems and that enables it to easily adapt to different numerical optimization problems. In [35], BSA was statistically compared with PSO, artificial bee colony algorithm (ABC), covariance matrix adaptation evolution strategy (CMAES), adaptive differential evolution algorithm (JDE), comprehensive learning particle swarm optimizer (CLPSO), and self-adaptive differential evolution algorithm (SADE). 75 boundary-constrained and three real-world benchmark problems were employed for the comparisons. In general, the simulations and comparisons in [35] showed that BSA can solve the benchmark problems more successfully than the compared algorithms. BSA was also used in [38] for circular antenna array design.

The rest of the paper is organized as follows. In Section 2, the general problem formulations for the synthesis of CCAA are given. A detailed explanation for the BSA is presented in Section 3. Numerical results are given in Section 4. Finally the conclusion of the paper is presented in Section 5.

\section{Problem Formulation}

Let us assume that a number of isotropic elements are placed in multiple concentric circular rings which differ in radius as illustrated in Figure 1. There are $M$ concentric circular rings in $x-y$ plane and the $m$ th ring has a radius $r_{m}$ and the number of elements is $N_{m}$, where $m=1,2, \ldots, M$. If the array elements are equally spaced within the ring, the azimuth angle from the $x$-axis for $m n$th element is given by

$$
\phi_{m n}=\frac{2 \pi n}{N_{m}}, \quad n=1,2,3, \ldots, N_{m}
$$

Thus, the array factor of the concentric circular antenna array can be formulated as follows:

$$
\operatorname{AF}(\phi)=I_{c}+\sum_{m=1}^{M} \sum_{n=1}^{N} I_{m n} e^{j\left(k r_{m}\left[\cos \left(\phi-\phi_{m n}\right)-\cos \left(\phi_{0}-\phi_{m n}\right)\right]\right)}
$$

where $I_{m n}$ is the excitation amplitude of the $n$th element in the $m$ th ring and $I_{c}$ is the excitation amplitude of the central element placed at the origin. In this paper, $\phi_{0}$ is fixed to 0 for all optimization examples in order to direct the peak of the main beam to the $x$ direction.

In this paper, we consider designing antenna arrays with the low maximum sidelobe level (MSL) at a fixed first null beamwidth (FNBW). Dynamic range ratio (DRR) is also considered in this paper since it is important for some practical considerations. Additionally, null steering on the sidelobe region is also studied in this paper. Thus, the following cost function is used:

$$
\begin{aligned}
C= & w_{\mathrm{MSL}} \cdot f_{\mathrm{MSL}}+w_{\mathrm{FNBW}} \cdot f_{\mathrm{FNBW}} \\
& +w_{\mathrm{DRR}} \cdot f_{\mathrm{DRR}}+w_{\mathrm{NULL}} \cdot f_{\mathrm{NULL}}
\end{aligned}
$$

where $w_{\mathrm{MSL}}, w_{\mathrm{FNBW}}, w_{\mathrm{DRR}}$, and $w_{\mathrm{NULL}}$ are the weight factors. $f_{\mathrm{MSL}}, f_{\mathrm{FNBW}}, f_{\mathrm{DRR}}$, and $f_{\mathrm{NULL}}$ are the functions used for suppressing MSL, fixing FNBW, decreasing DRR, and locating nulls, respectively. The $f_{\mathrm{MSL}}$ function can be given as follows:

$$
f_{\mathrm{MSL}}=\int_{-\pi}^{\phi_{\text {null } 1}} \sigma_{\mathrm{MSL}}(\phi) d \phi+\int_{\phi_{\text {null }}}^{\pi} \sigma_{\mathrm{MSL}}(\phi) d \phi,
$$

where $\phi_{\text {null1 }}$ and $\phi_{\text {null2 }}$ are the two angles at the first nulls on each side of the main beam. $\sigma_{\mathrm{MSL}}(\phi)$ can be formulated as follows:

$$
\sigma_{\mathrm{MSL}}(\phi)= \begin{cases}\psi_{o}(\phi)-\mathrm{MSL}_{d}, & \psi_{o}(\phi)>\mathrm{MSL}_{d} \\ 0, & \text { elsewhere, }\end{cases}
$$

where $\psi_{o}(\phi)$ is the array factor in $\mathrm{dB}$ and $\mathrm{MSL}_{d}$ is the desired MSL value. The function $f_{\mathrm{FNBW}}$ in (3) can be given by

$$
f_{\mathrm{FNBW}}= \begin{cases}\mathrm{FNBW}_{o}-f_{\text {fnbwmax }}, & \mathrm{FNBW}_{o}>f_{\text {fnbwmax }} \\ 0, & \text { elsewhere, }\end{cases}
$$

where $\mathrm{FNBW}_{o}$ and $f_{\text {fnbwmax }}$ are the FNBW value obtained by BSA and the desired maximum FNBW, respectively. The function $f_{\mathrm{DRR}}$ can be given by

$$
f_{\mathrm{DRR}}= \begin{cases}\left|\frac{I_{\mathrm{max}}}{I_{\min }}\right|-f_{\mathrm{drrmax}}, & \left|\frac{I_{\max }}{I_{\min }}\right|>f_{\mathrm{drrmax}} \\ 0, & \text { elsewhere }\end{cases}
$$

where $I_{\max }$ and $I_{\min }$ are the maximum and minimum amplitude values of the antenna array elements obtained by the BSA, respectively. $f_{\text {drrmax }}$ is the desired maximum DRR value. In a similar manner, $f_{\mathrm{NULL}}$ is given as follows:

$$
f_{\mathrm{NULL}}= \begin{cases}\left(\mathrm{NDL}_{o}-\mathrm{NDL}_{d}\right)^{2}, & \text { if } \mathrm{NDL}_{o}>\mathrm{NDL}_{d} \\ 0, & \text { elsewhere. }\end{cases}
$$

$\mathrm{NDL}_{o}$ and $\mathrm{NDL}_{d}$ are the obtained and desired maximum null depth level (NDL) values, respectively. In this way, the cost function can manage the MSL, FNBW, DRR, and NDL values.

\section{Backtracking Search Optimization Algorithm (BSA)}

BSA [35] is one of the new population based evolutionary algorithms. It is based on an iterative process which tries to minimize the objective function. BSA consists of five evolutionary mechanisms: initialization, selection-I, mutation, crossover, and selection-II. The general flowchart of BSA is illustrated in Figure 2.

3.1. Initialization. BSA initially scatters the population members in the solution space by using a uniform random distribution function:

$$
P_{i, j} \sim U\left(\operatorname{low}_{j}, \operatorname{up}_{j}\right), \quad i=1,2,3, \ldots, N, j=1,2,3, \ldots, D,
$$

where $N$ and $D$ are the population size and the problem dimension, respectively. $U$ is the uniform distribution function and $P_{i}$ is the position of the $i$ th population member in the solution space. low $_{j}$ and $\mathrm{up}_{j}$ in (9) are lower and upper bounds of solution space, respectively. 


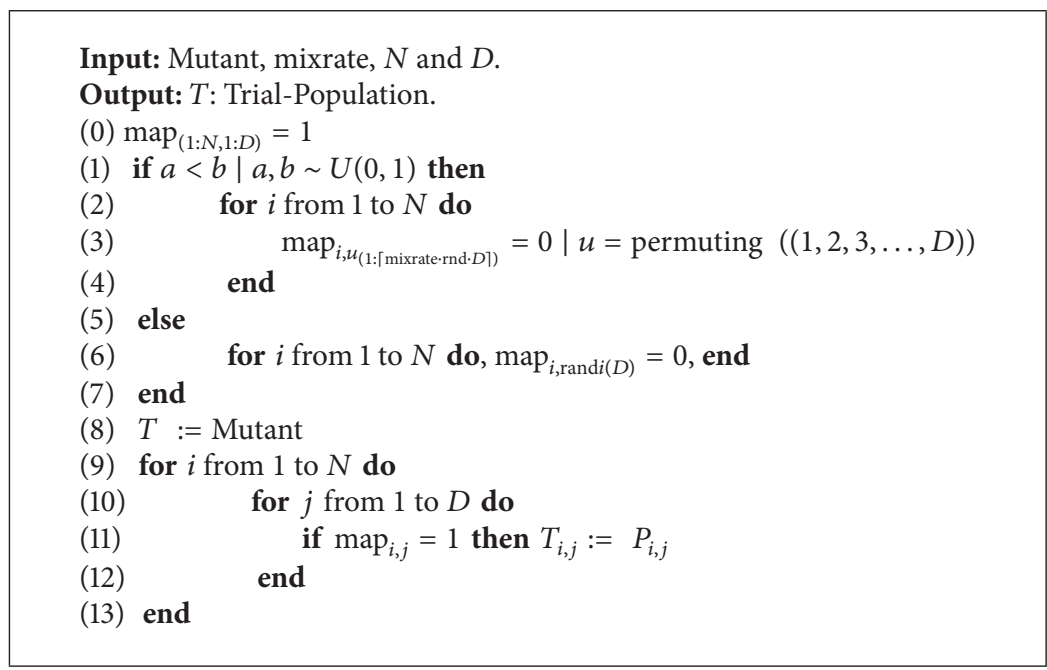

Algorithm 1: Crossover strategy of BSA.

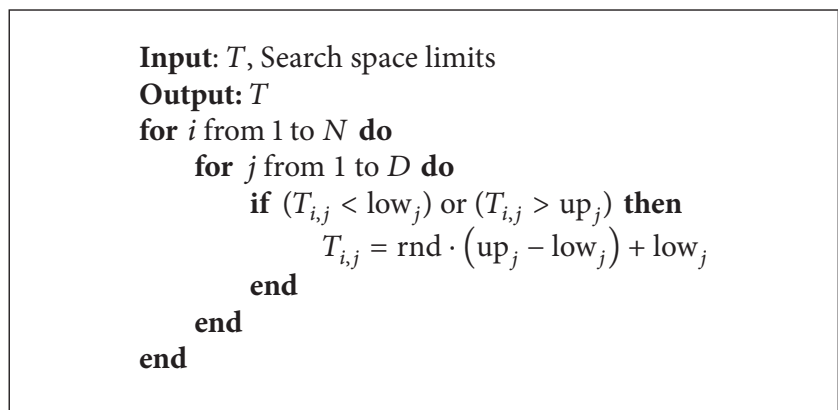

Algorithm 2: Boundary control mechanism of BSA.

3.2. Selection-I. In the Selection-I stage, BSA generates the historical population utilized to determine the search direction. The following equation is used to initialize the historical population:

$$
\operatorname{oldP}_{i, j} \sim U\left(\operatorname{low}_{j}, \mathrm{up}_{j}\right) .
$$

The following equation gives a chance to BSA to redesign oldP at the beginning of each iteration:

$$
\text { if } a<b \text { then oldP }:=P \mid a, b \sim U(0,1),
$$

where $a$ and $b$ are the uniform real numbers in the range of $[0,1]$ in order to decide if the oldP is selected from previous generation. The following equation is used for shuffling the order of the population members:

$$
\text { old } P:=\text { permuting }(\text { old } P),
$$

where the permuting( ) function is a random shuffling function.

3.3. Mutation. The mutant members of BSA are generated by using the following function:

$$
\text { Mutant }=P+F \cdot(\text { old } P-P),
$$

where $F$ is a real number to control the step size amplification of the search direction. BSA employed historical data to determine the search direction of the population members by taking into account oldP values.

3.4. Crossover. The final form of the trial-population $T$ is generated by the crossover process of the BSA. The crossover process of the BSA is given in a pseudocode form in Algorithm 1. The crossover step includes two steps. The first strategy uses mixrate (Algorithm 1, lines 2-4). The second strategy allows only one randomly chosen individual to mutate in each trial (Algorithm 1, line 6). After the crossover process, the individuals may exceed the search space boundaries. In order to restrict the movement area of the individuals, the procedure given in Algorithm 2 is activated when needed. The restriction process provides a random regeneration in the valid search space for the individuals which exceed the boundaries.

3.5. Selection-II. In selection-II process of BSA, $P_{i} s$ values are updated by a greedy selection strategy that uses $T_{i} s$ which have better fitness than the corresponding $P_{i} s$ values. Global minimum value is replaced by the best individual of $P\left(P_{\text {best }}\right)$ 


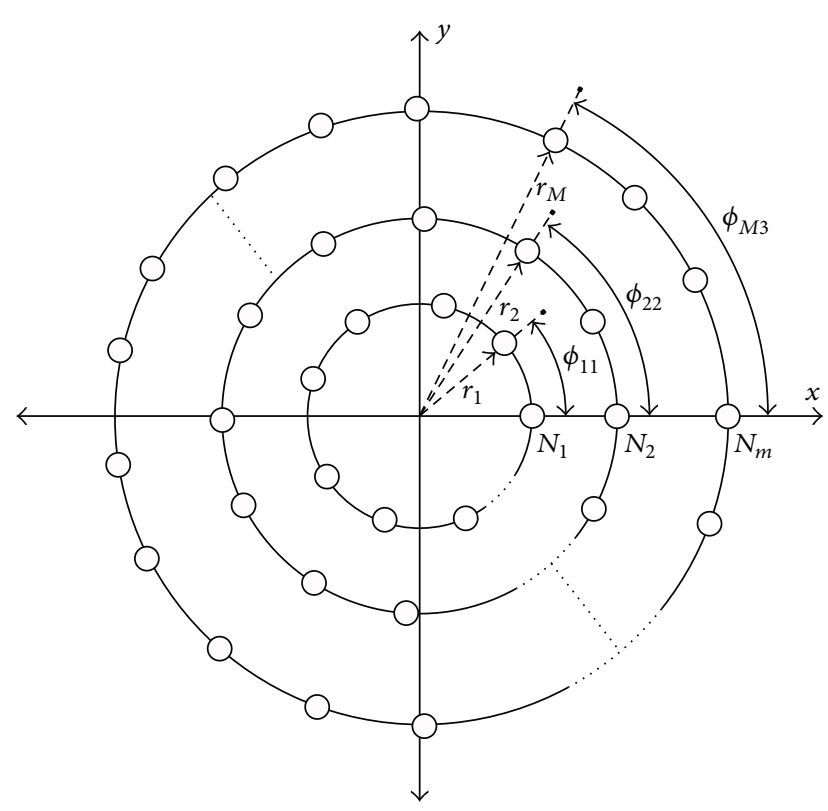

FIGURE 1: Geometry of a concentric circular array.

TABLE 1: MSL, FNBW, and DRR values obtained by BSA and other optimization techniques EP [1], PSO [1], PSOCFIWA [1], CPSO [2], RGA [2], CRPSO [2], HEP [2], BFO [3], and BGA [3] for CCAAs without central elements.

\begin{tabular}{lcccc}
\hline Figures & Algorithms & MSL (dB) & FNBW (degree) & DRR \\
\hline \multirow{2}{*}{ Figure 3 } & BSA & -33.74 & 76.36 & 10.2775 \\
& EP [1] & -31.90 & 78.50 & 18.8021 \\
\hline \multirow{2}{*}{ Figure 4 } & BSA & -29.49 & 71.79 & 13.6986 \\
& PSO [1] & -27.18 & 72.24 & Inf. \\
\hline \multirow{2}{*}{ Figure 5 } & BSA & -30.60 & 76.45 & 3.5063 \\
& PSOCFIWA [1] & -28.48 & 78.10 & 3.6062 \\
\hline \multirow{2}{*}{ Figure 6 } & BSA & -31.65 & 74.56 & 4.6577 \\
& CPSO [2] & -28.96 & 76.30 & 4.8876 \\
\hline \multirow{2}{*}{ Figure 7 } & BSA & -31.00 & 77.00 & 3.4374 \\
& RGA [2] & -28.06 & 77.10 & 3.4435 \\
\hline \multirow{2}{*}{ Figure 8 } & BSA & -32.15 & 76.00 & 4.1999 \\
& CRPSO [2] & -29.88 & 76.40 & 4.4309 \\
\hline \multirow{2}{*}{ Figure 9 } & BSA & -33.71 & 76.26 & 8.2850 \\
& HEP [2] & -32.86 & 77.00 & 11.6875 \\
\hline \multirow{2}{*}{ Figure 10 } & BSA & -32.51 & 75.30 & 4.8008 \\
& BFO [3] & -29.96 & 75.80 & 4.8112 \\
\hline \multirow{2}{*}{ Figure 11 } & BSA & -30.02 & 72.64 & 5.1706 \\
& BGA [3] & -26.14 & 73.60 & 5.3419 \\
\hline
\end{tabular}

when $P_{\text {best }}$ value is better than global minimum value. The detailed descriptions of BSA can be found in [35].

\section{Numerical Results}

In this paper, a CCAA which has three rings is synthesized by using BSA for all examples. The number of innermost, middle, and outermost rings has 4,6 , and 8 array elements,

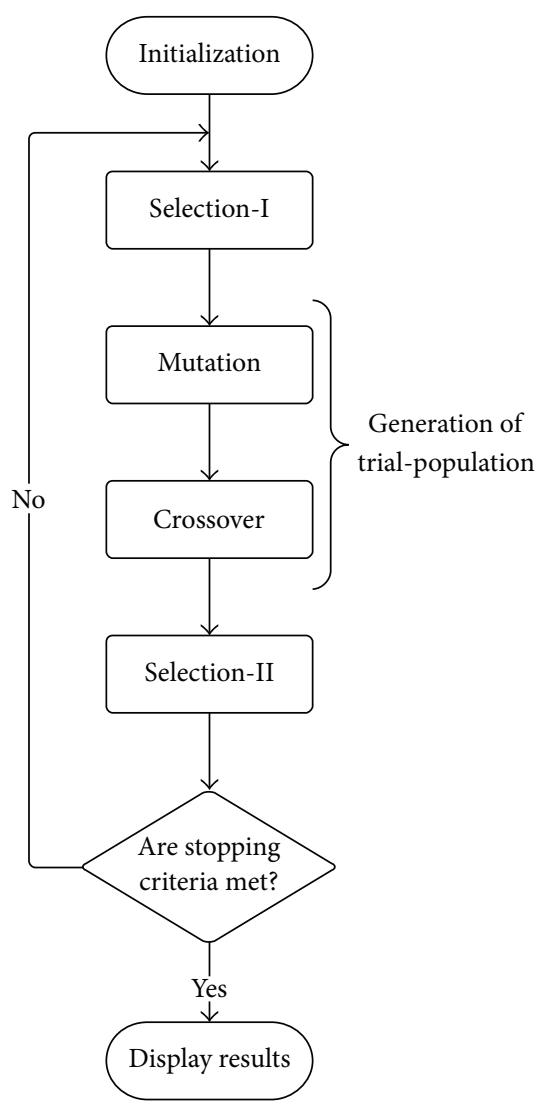

FIgURE 2: General flowchart of BSA.

respectively. Additionally, CCAA with a central element is also considered. The main goal is to obtain array patterns having low MSL and narrow FNBW values. DRR is also taken into account for the practical considerations. In order to test the flexibility of the algorithm, single, double, triple, and wide nulls on the sidelobe region of the pattern are also located by using BSA. The distances between two elements in the innermost, middle, and outermost rings are $0.55 \lambda, 0.606 \lambda$, and $0.75 \lambda$, respectively. The population sizes of BSA for the all examples in this section are set to 30 . The maximum iteration number is selected as 5000 . The special parameter of BSA mixrate is fixed to 1 . Step size amplification is determined as $F=3 \cdot$ rndn, where rndn $\sim N(0,1)$. The optimization processes are carried on a computer which has $2.8 \mathrm{GHz}$ i7 processor and 4GB RAM. The software used for the optimization implementations of BSA is MATLAB.

To show the performance and flexibility of the proposed BSA on the CCAA pattern synthesis, comprehensive comparisons have been made in this paper. In these comparisons, the results of BSA are compared with the results of nine different algorithms from the literature [1-3]. These algorithms are EP [1], PSO [1], PSO with constriction factor and inertia weight approach (PSOCFIWA) [1], canonical PSO (CPSO) [2], real coded genetic algorithm (RGA) [2], craziness based PSO (CRPSO) [2], hybrid evolutionary programming (HEP) [2], $\mathrm{BFO}$ [3], and binary coded genetic algorithm (BGA) [3]. In order to properly compare the results of BSA with the results 
TABLE 2: The amplitude values calculated by BSA for CCAAs without central element.

\begin{tabular}{|c|c|c|c|c|c|c|c|c|c|}
\hline \multirow{3}{*}{$\begin{array}{l}\text { Figures } \\
\text { Figure } 3\end{array}$} & \multicolumn{9}{|c|}{$I_{11}, I_{12}, I_{13}, \ldots, I_{1 m}$} \\
\hline & 0.1040 & 0.6725 & 0.0973 & 0.6684 & 0.4152 & 0.4148 & 0.9981 & 0.4169 & 0.4174 \\
\hline & 1.0000 & 0.4878 & 0.7053 & 0.4874 & 0.2360 & 0.4903 & 0.6984 & 0.4907 & 0.2361 \\
\hline \multirow{2}{*}{ Figure 4} & 0.0781 & 0.4107 & 0.0730 & 0.4994 & 0.3233 & 0.3093 & 0.9764 & 0.3223 & 0.3400 \\
\hline & 1.0000 & 0.5015 & 0.6399 & 0.4534 & 0.1616 & 0.4278 & 0.6272 & 0.4793 & 0.1639 \\
\hline \multirow{2}{*}{ Figure 5} & 0.2852 & 0.7856 & 0.2852 & 0.7476 & 0.4960 & 0.5103 & 1.0000 & 0.4939 & 0.5009 \\
\hline & 1.0000 & 0.5051 & 0.7743 & 0.4738 & 0.2852 & 0.5366 & 0.7876 & 0.4881 & 0.2852 \\
\hline \multirow{2}{*}{ Figure 6} & 0.2147 & 0.6903 & 0.2147 & 0.7269 & 0.4364 & 0.4331 & 1.0000 & 0.4444 & 0.4373 \\
\hline & 1.0000 & 0.5113 & 0.7518 & 0.5219 & 0.2147 & 0.4923 & 0.7815 & 0.5063 & 0.2147 \\
\hline \multirow{2}{*}{ Figure 7} & 0.2771 & 0.7591 & 0.2772 & 0.7643 & 0.4932 & 0.4922 & 0.9525 & 0.4923 & 0.4933 \\
\hline & 0.9525 & 0.4695 & 0.7605 & 0.4684 & 0.2771 & 0.4701 & 0.7618 & 0.4712 & 0.2771 \\
\hline \multirow{2}{*}{ Figure 8} & 0.2381 & 0.7398 & 0.2381 & 0.7469 & 0.4688 & 0.4630 & 1.0000 & 0.4659 & 0.4716 \\
\hline & 1.0000 & 0.4978 & 0.7682 & 0.4979 & 0.2408 & 0.4988 & 0.7737 & 0.4987 & 0.2381 \\
\hline \multirow{2}{*}{ Figure 9} & 0.1207 & 0.6684 & 0.1207 & 0.6819 & 0.4195 & 0.4125 & 1.0000 & 0.4126 & 0.4196 \\
\hline & 0.9985 & 0.4844 & 0.7186 & 0.4867 & 0.2371 & 0.4868 & 0.7177 & 0.4844 & 0.2328 \\
\hline \multirow{2}{*}{ Figure 10} & 0.2083 & 0.7207 & 0.2083 & 0.7074 & 0.4338 & 0.4440 & 1.0000 & 0.4436 & 0.4333 \\
\hline & 1.0000 & 0.5027 & 0.7597 & 0.5001 & 0.2104 & 0.4996 & 0.7581 & 0.5022 & 0.2208 \\
\hline \multirow{2}{*}{ Figure 11} & 0.1934 & 0.6853 & 0.1934 & 0.6859 & 0.4335 & 0.4330 & 1.0000 & 0.4321 & 0.4326 \\
\hline & 1.0000 & 0.4947 & 0.7988 & 0.4946 & 0.1934 & 0.4957 & 0.7976 & 0.4958 & 0.1934 \\
\hline
\end{tabular}

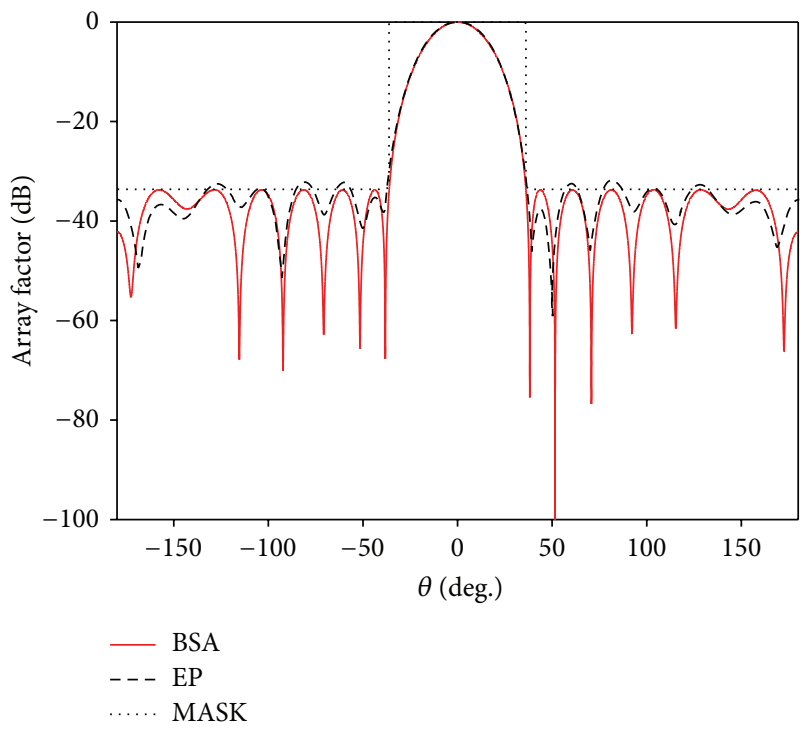

FIGURE 3: The radiation patterns obtained by BSA and EP [1] for CCAA without central element.

of the other nine algorithms [1-3], the desired MSL, FNBW, and DRR values are chosen to be similar to those of nine algorithms presented in [1-3].

The CCAA considered for the first group of examples does not have a central array element. BSA is employed to achieve a pattern which has low MSL and narrow FNBW values. The DRR values of the CCAAs are also considered in the cost function in the same optimization process. The array patterns obtained by BSA used in this paper are compared with the patterns of EP [1], PSO [1], PSOCFIWA [1], CPSO [2], RGA [2], CRPSO [2], HEP [2], BFO [3], and BGA [3] in

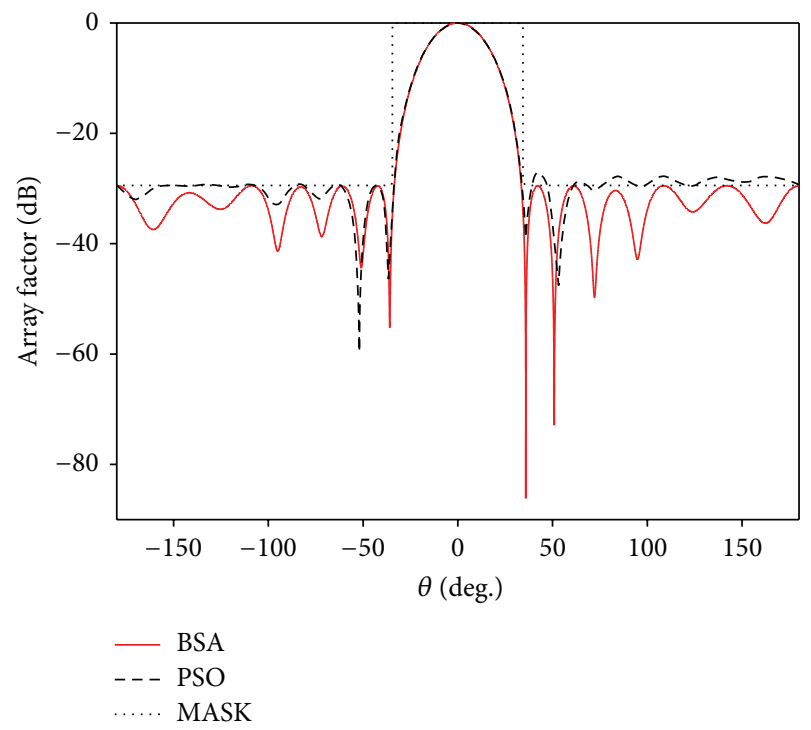

FIGURE 4: The radiation patterns obtained by BSA and PSO [1] for CCAA without central element.

Figures 3-11, respectively. The MSL, FNBW, and DRR values obtained by BSA and the other nine algorithms are given in Table 1. It is apparent from Figures 3-11 and Table 1 that MSL, FNBW, and DRR values of the patterns produced by using BSA are better than those of EP [1], PSO [1], PSOCFIWA [1], CPSO [2], RGA [2], CRPSO [2], HEP [2], BFO [3], and BGA [3]. The amplitude values calculated by BSA for the patterns presented in Figures 3-11 are given in Table 2.

Moreover, the convergence curve of BSA is compared with those of DS [36] and BFA [37] for the first example represented by Figure 3. The maximum iteration number and 


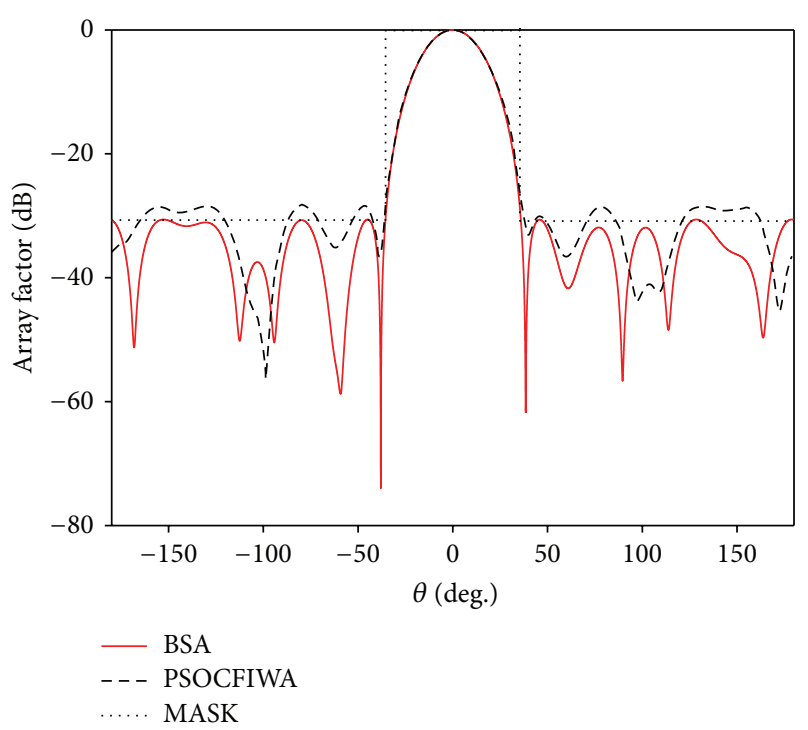

FIGURE 5: The radiation patterns obtained by BSA and PSOCFIWA [1] for CCAA without central element.

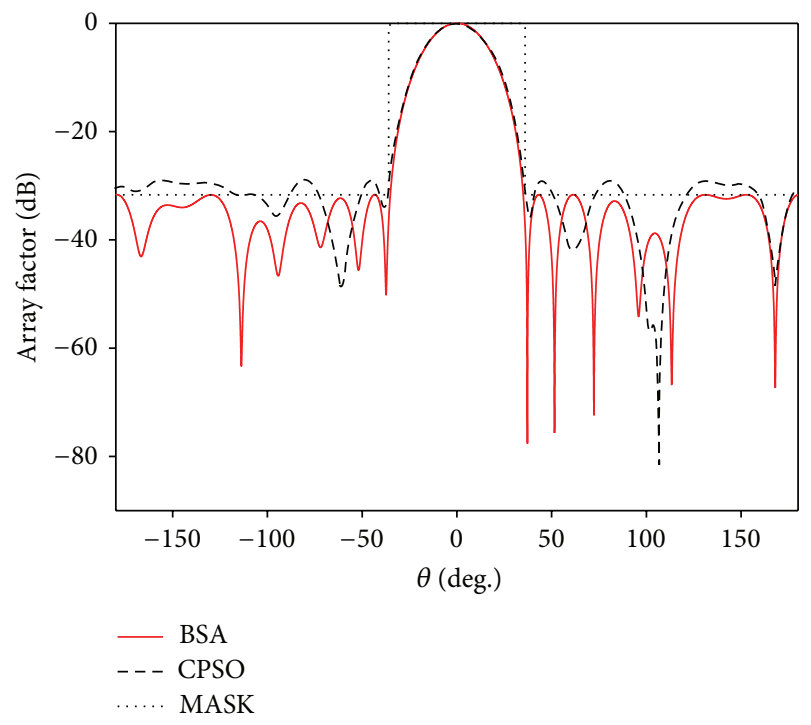

FIGURE 6: The radiation patterns obtained by BSA and CPSO [2] for CCAA without central element.

the population number for all three algorithms are 700 and 50 , respectively. Whereas DS algorithm does not need any extra parameter definition, the algorithm parameters of BFA are set as follows: number of chemotactic steps is 175, length of swim is 4 , number of reproduction steps is 4 , number of elimination-dispersal events is 1 , and length of run is 0.4. Figure 12 shows the convergence curves of BSA, DS, and BFA for the first example in the first group of examples. From Figure 12, it is clearly seen that BSA can converge the better cost function value at the end of the iteration than DS and BFA.

For the second group of examples, the CCAA with central array element is considered. Figures 13-17 show the patterns

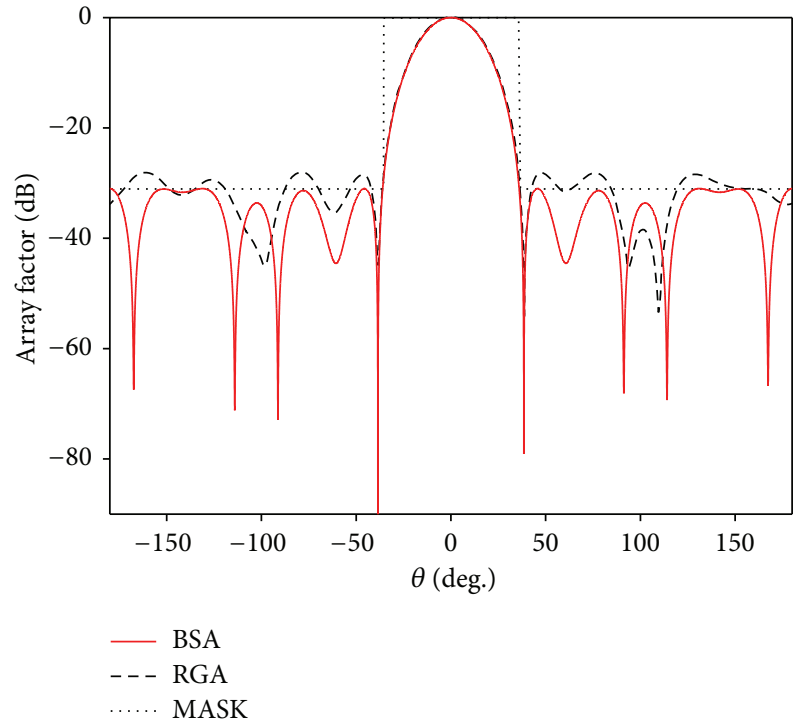

FIGURE 7: The radiation patterns obtained by BSA and RGA [2] for CCAA without central element.

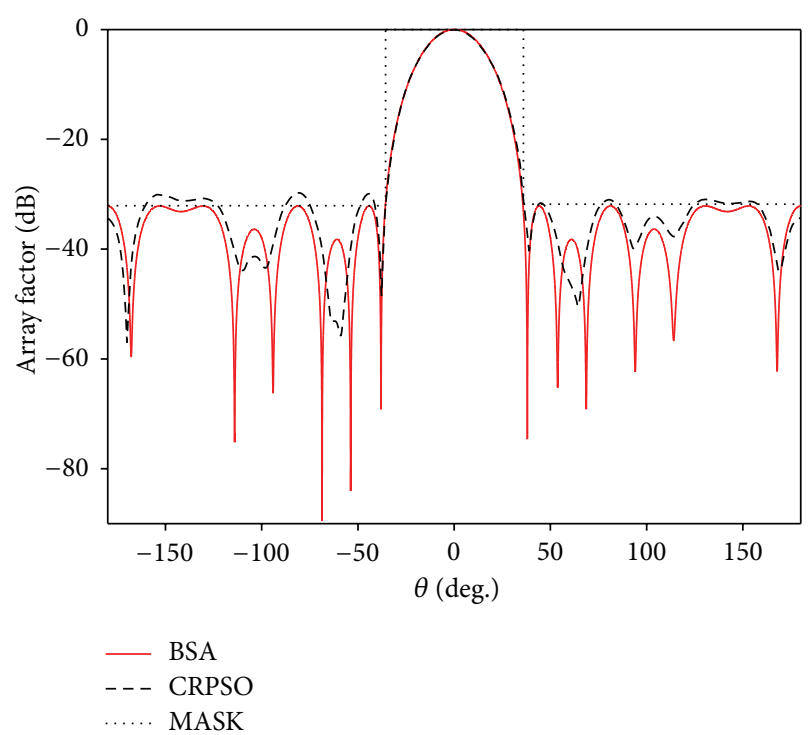

FIGURE 8: The radiation patterns obtained by BSA and CRPSO [2] for CCAA without central element.

obtained by BSA. For a comparison, the patterns achieved by EP [1], PSOCFIWA [1], PSO [1], BFO [3], and BGA [3] are also included in these figures. The MSL, FNBW, and DRR values obtained by BSA are compared with those of EP [1], PSOCFIWA [1], PSO [1], BFO [3], and BGA [3] in Table 3. As it can be seen from Figures 13-17 and Table 3, all MSL and FNBW values achieved by BSA are better than those of the compared algorithms in [1] and [3]. Additionally, the DRR values calculated by BSA are better than those of EP [1], PSOCFIWA [1], BFO [3], and BGA [3]. On the other hand, the DRR value obtained by BSA used in this paper is the same with that of PSO [1]. The amplitude values calculated 


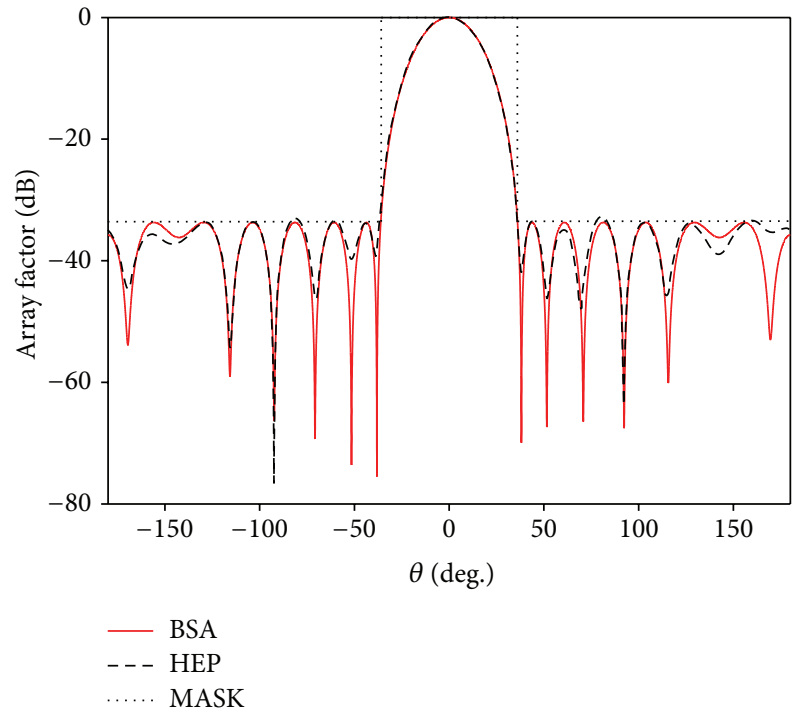

FIGURE 9: The radiation patterns obtained by BSA and HEP [2] for CCAA without central element.

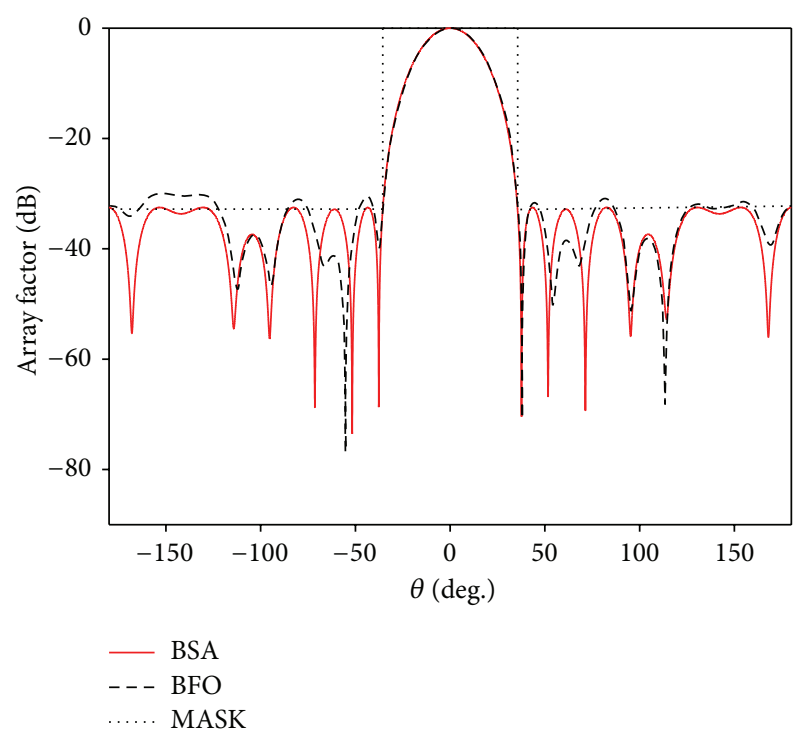

FIGURE 10: The radiation patterns obtained by BSA and BFO [3] for CCAA without central element.

by BSA for the patterns presented in Figures 13-17 are given in Table 4.

In the last group of examples, four synthesis examples are studied in order to examine the capability of BSA for steering single, multiple, and wide nulls on the sidelobe region of CCAA pattern. The amplitude values of array elements are controlled by BSA. A CCAA pattern with a single null imposed at $70^{\circ}$ is considered as the first pattern nulling example. Figure 18 shows the radiation pattern obtained by BSA. From Figure 18, it can be seen that NDL value at $70^{\circ}$ is 106.9 dB and MSL value is $-33 \mathrm{~dB}$. For the second and third

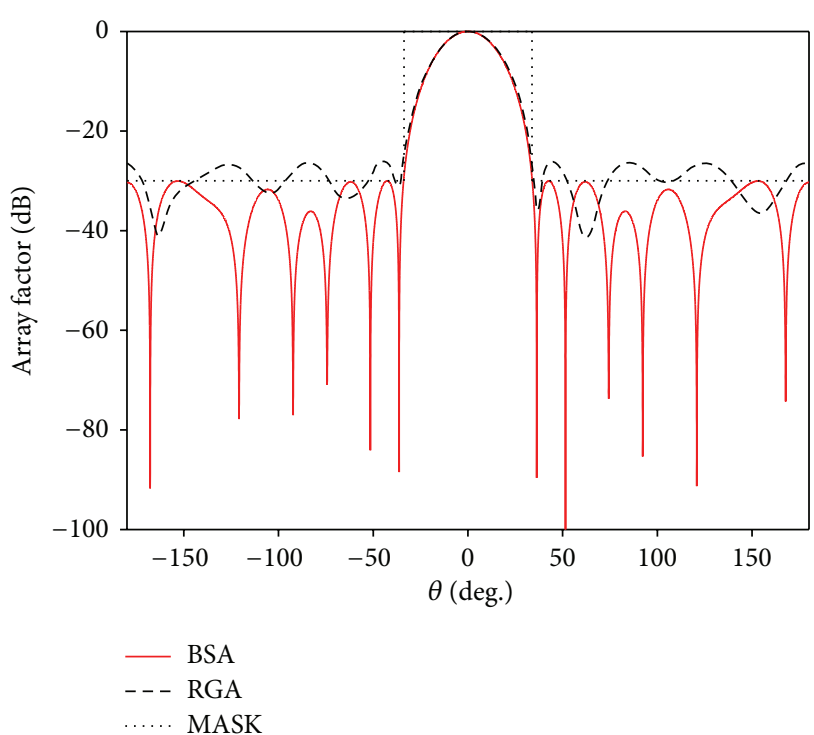

FIGURE 11: The radiation patterns obtained by BSA and BGA [3] for CCAA without central element.

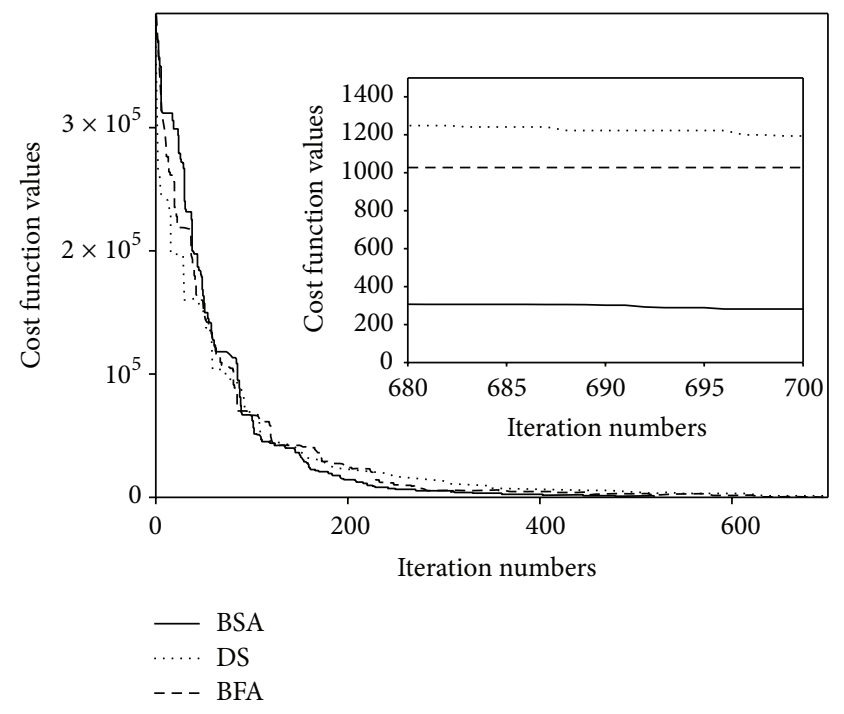

FIGURE 12: The convergence curves of BSA, DS, and BFA.

pattern nulling examples, double $\left(70^{\circ}\right.$ and $\left.108^{\circ}\right)$ and triple $\left(53^{\circ}, 70^{\circ}\right.$, and $\left.109^{\circ}\right)$ nulls are placed by using BSA on the sidelobe region of the CCAA pattern. Figures 19 and 20 show the patterns having double and triple nulls, respectively. For both examples, all NDL values are deeper than $-85 \mathrm{~dB}$ and all MSL values are less than $-30 \mathrm{~dB}$. In the last example, a CCAA pattern with wide null at $75^{\circ}$ with $\Delta \theta=10^{\circ}$ is synthesized by using BSA. Figure 21 illustrates the pattern achieved by BSA. From Figure 21, it can be seen that NDL value is $-62 \mathrm{~dB}$ and MSL value is $-30.1 \mathrm{~dB}$. The amplitude values calculated by BSA for the patterns presented in Figures 18-21 are given in Table 5.

From Figures 3-11 and 13-17, it can be concluded that BSA is capable of synthesizing CCAA patterns with low 


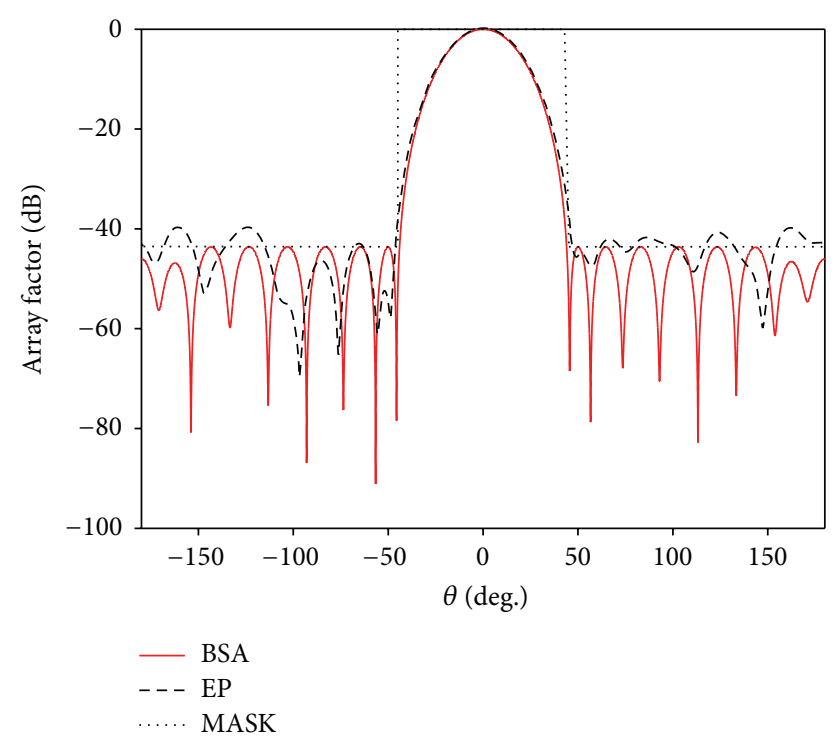

FIGURE 13: The radiation patterns obtained by BSA and EP [1] for CCAA with central element.

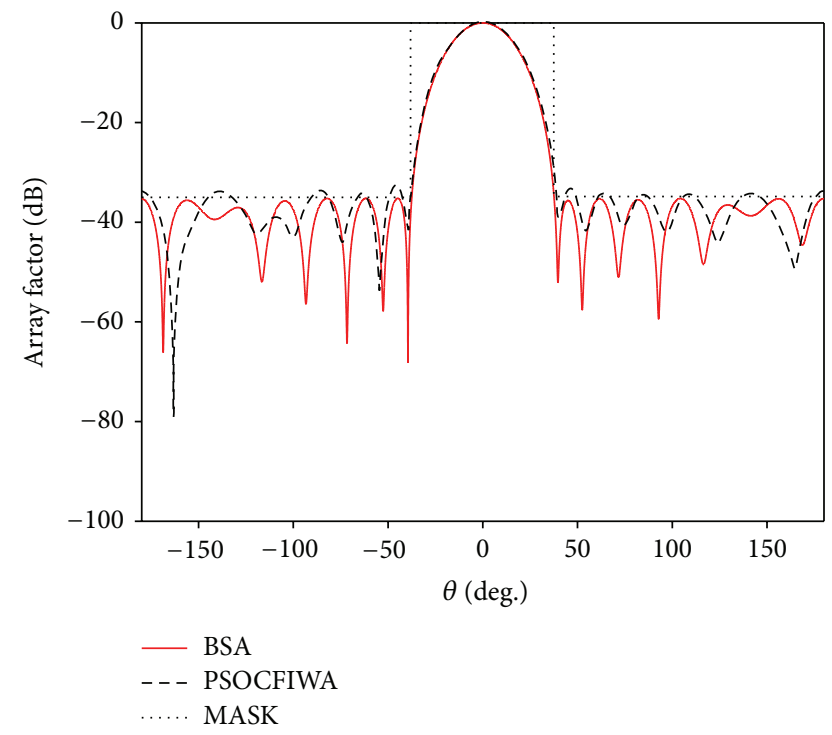

FIGURE 14: The radiation patterns obtained by BSA and PSOCFIWA [1] for CCAA with central element.

MSL and narrow FNBW values. Besides, BSA can be used to achieve low DRR values within the same optimization process. Furthermore, according to Figures 18-21, it can be said that BSA has capacity to synthesize the CCAA patterns with low MSL values and single, double, triple, and wide nulls at the desired directions.

\section{Conclusion}

In this paper, BSA is used for the synthesis of CCAA patterns with the low sidelobe levels at a fixed beamwidth and patterns with the single, multiple, and broad nulls imposed at the

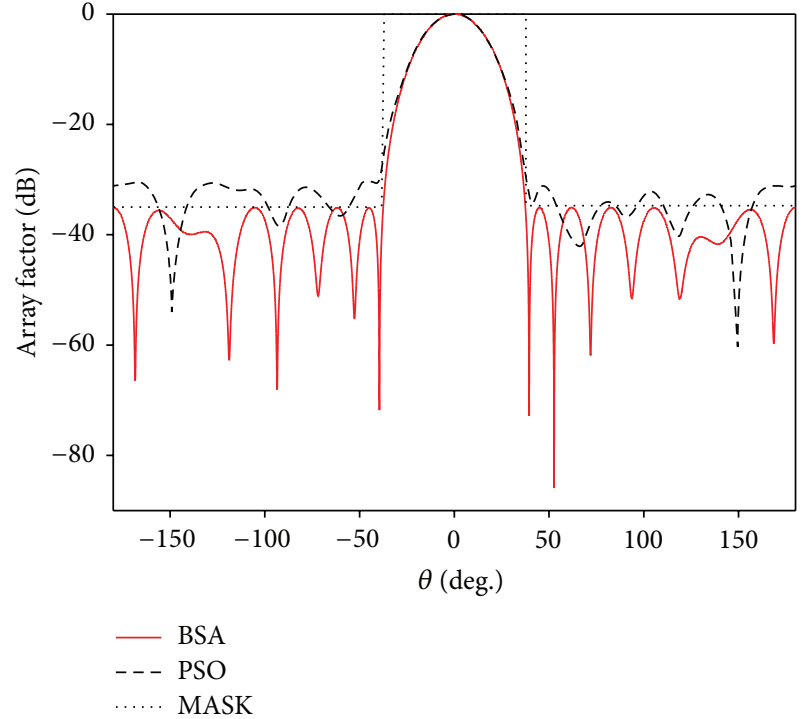

FIGURE 15: The radiation patterns obtained by BSA and PSO [1] for CCAA with central element.

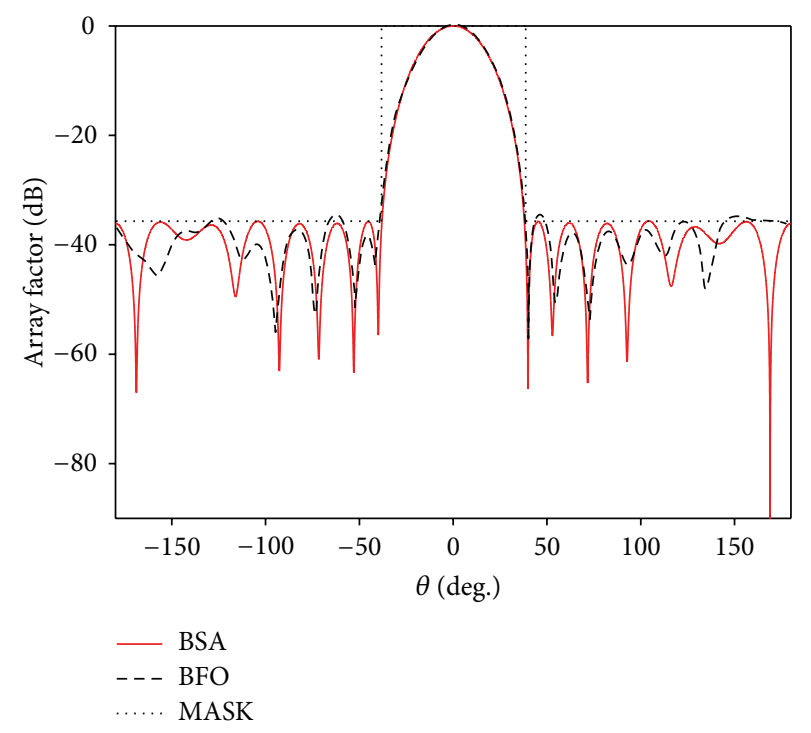

FIGURE 16: The radiation patterns obtained by BSA and BFO [3] for CCAA with central element.

directions of interference. It can be concluded that the design of CCAA using BSA provides good sidelobe levels and null depth levels with a fixed beamwidth. The comparisons made between the results obtained by BSA and several metaheuristic algorithms show that BSA is a very competitive algorithm for the synthesis of antenna array patterns. Additionally, the convergence curve comparison shows that BSA has better convergence characteristic than DS and BFA. 


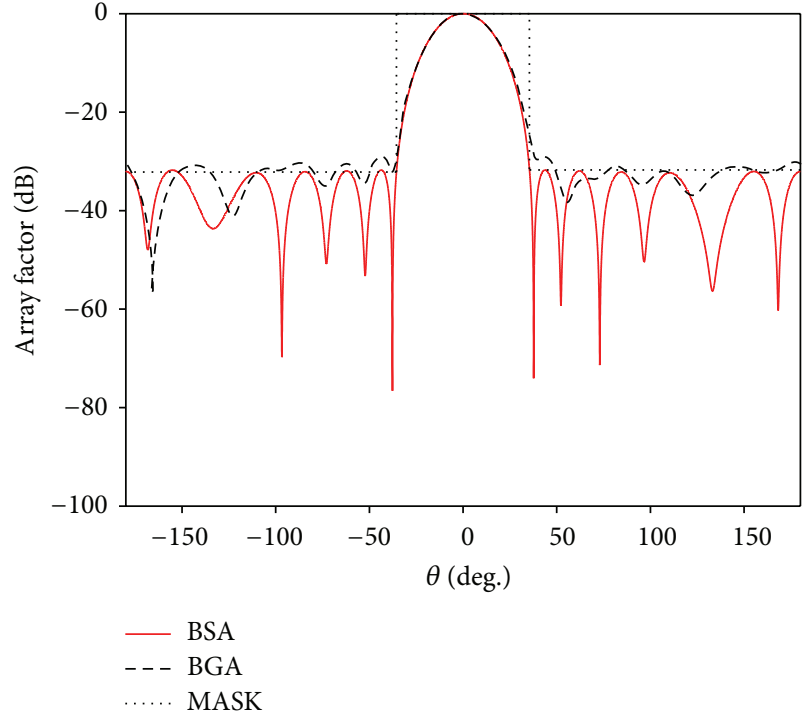

FIGURE 17: The radiation patterns obtained by BSA and BGA [3] for CCAA with central element.

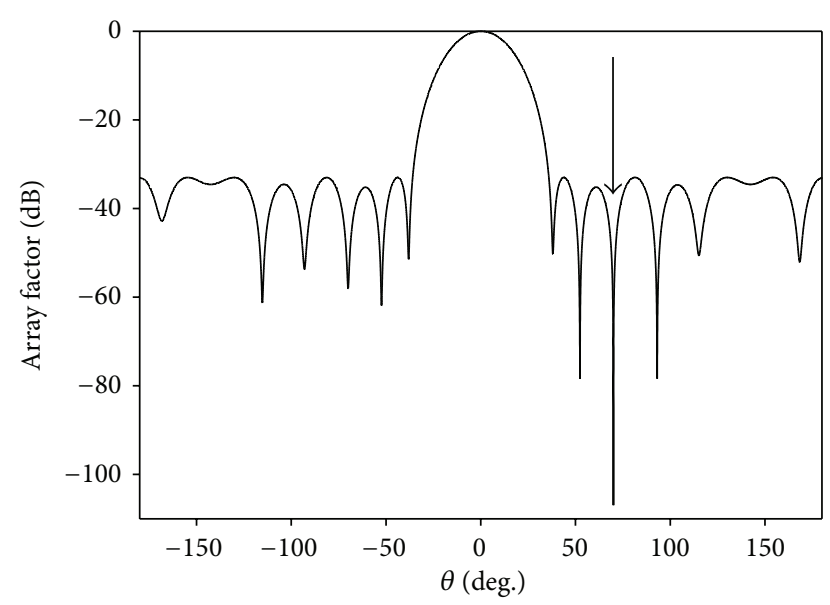

FIGURE 18: Radiation pattern obtained by amplitude-only control with one imposed null at $70^{\circ}$.

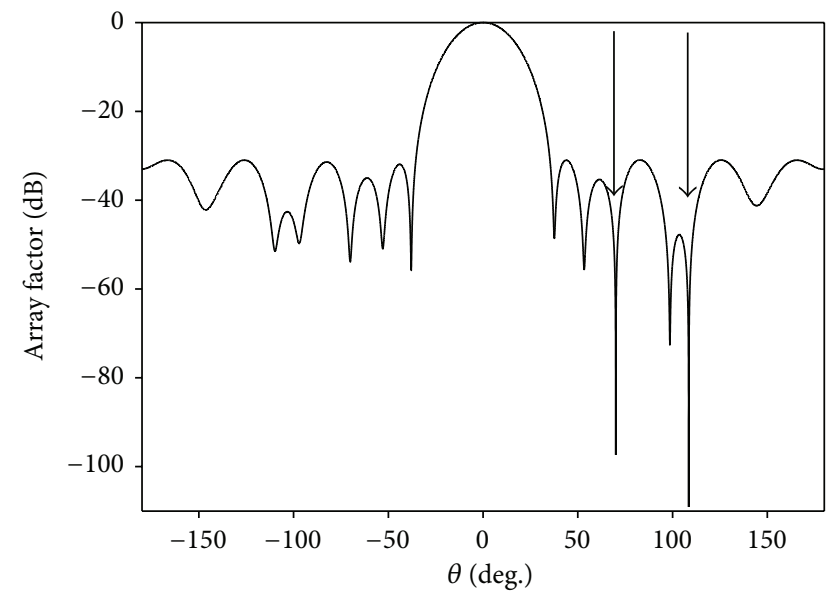

FIGURE 19: Radiation pattern obtained by amplitude-only control with double imposed null at $70^{\circ}$ and $108^{\circ}$.

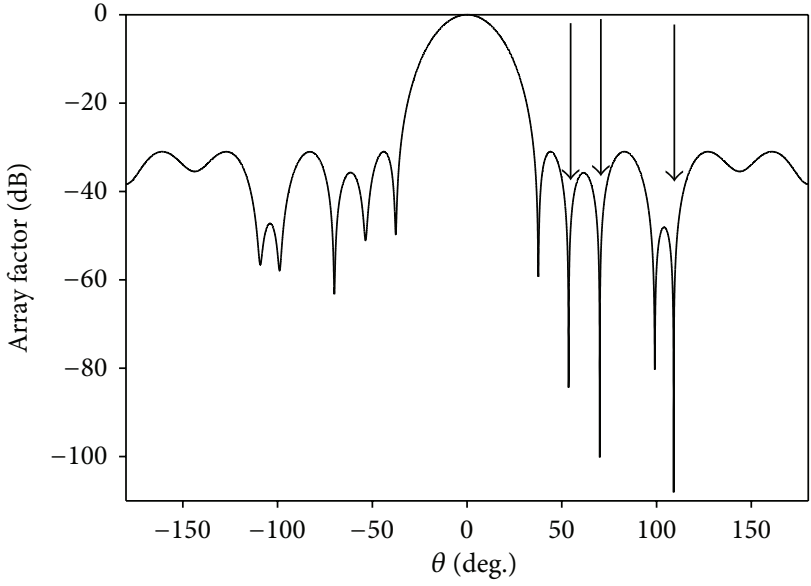

FIGURE 20: Radiation pattern obtained by amplitude-only control with triple imposed null at $53^{\circ}, 70^{\circ}$, and $109^{\circ}$.

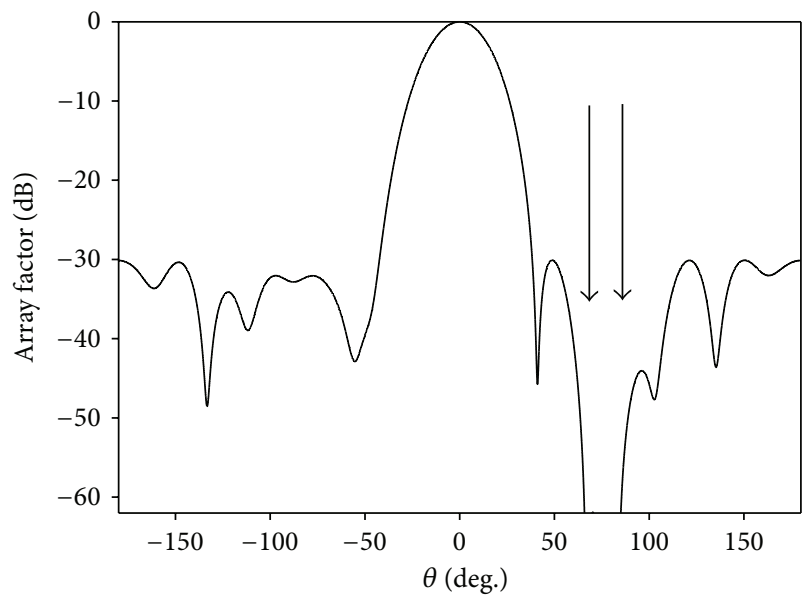

FIGURE 21: Radiation pattern obtained by amplitude-only control with a broad null sector centered $75^{\circ}$ with $\Delta \theta=10^{\circ}$.

TABLE 3: MSLL, FNBW, and DRR values obtained by BSA and other optimization techniques EP [1], PSOCFIWA [1], PSO [1], BFO [3], and BGA [3] for CCAAs with central element.

\begin{tabular}{lcccc}
\hline Figures & Algorithms & MSL (dB) & FNBW (degree) & DRR \\
\hline \multirow{2}{*}{ Figure 13 } & BSA & -43.61 & 91.20 & 9.1827 \\
& EP [1] & -39.66 & 97.70 & 14.6883 \\
\hline \multirow{2}{*}{ Figure 14 } & BSA & -35.20 & 79.14 & 5.5928 \\
& PSOCFIWA [1] & -32.62 & 80.00 & Inf. \\
\hline \multirow{2}{*}{ Figure 15 } & BSA & -35.08 & 79.01 & 4.8054 \\
& PSO [1] & -30.08 & 82.37 & 4.8054 \\
\hline \multirow{2}{*}{ Figure 16 } & BSA & -35.89 & 79.89 & 4.5005 \\
& BFO [3] & -34.18 & 81.50 & 4.8869 \\
\hline \multirow{2}{*}{ Figure 17 } & BSA & -31.86 & 75.45 & 6.9589 \\
& BGA [3] & -29.06 & 78.30 & 7.0569 \\
\hline
\end{tabular}


TABLE 4: The amplitude values calculated by BSA for the CCAAs with central element.

\begin{tabular}{|c|c|c|c|c|c|c|c|c|c|}
\hline Figures & & & & & $I_{13}, \ldots$ & & & & \\
\hline & 0.9568 & 1.0000 & 0.9578 & 0.9943 & 0.7376 & 0.7409 & 0.3565 & 0.7384 & 0.7350 \\
\hline Figure 13 & 0.3638 & 0.3929 & 0.5322 & 0.3880 & 0.1089 & 0.3897 & 0.5327 & 0.3945 & 0.1116 \\
\hline & & & & & 0.6245 & & & & \\
\hline & 0.4145 & 0.9105 & 0.4301 & 0.8732 & 0.5958 & 0.6003 & 1.0000 & 0.5968 & 0.5827 \\
\hline Figure 14 & 0.9920 & 0.5586 & 0.8135 & 0.5638 & 0.2334 & 0.5498 & 0.8208 & 0.5350 & 0.2224 \\
\hline & & & & & 0.1788 & & & & \\
\hline & 0.4773 & 0.9295 & 0.4877 & 0.9271 & 0.6335 & 0.6372 & 1.0000 & 0.6261 & 0.6221 \\
\hline Figure 15 & 1.0000 & 0.5653 & 0.8490 & 0.5546 & 0.2081 & 0.5582 & 0.8393 & 0.5722 & 0.2238 \\
\hline & & & & & 0.2081 & & & & \\
\hline & 0.4941 & 0.9592 & 0.4931 & 0.9520 & 0.6350 & 0.6339 & 1.0000 & 0.6481 & 0.6484 \\
\hline Figure 16 & 0.9967 & 0.5606 & 0.8306 & 0.5669 & 0.2380 & 0.5807 & 0.8557 & 0.5763 & 0.2298 \\
\hline & & & & & 0.2222 & & & & \\
\hline & 0.4790 & 0.8495 & 0.5000 & 0.8854 & 0.6077 & 0.5984 & 0.9927 & 0.5936 & 0.6035 \\
\hline Figure 17 & 1.0000 & 0.5593 & 0.8764 & 0.5433 & 0.1437 & 0.5215 & 0.8736 & 0.5375 & 0.1445 \\
\hline & & & & & 0.1485 & & & & \\
\hline
\end{tabular}

TABLE 5: The amplitude values calculated by BSA for pattern nulling synthesis examples.

\begin{tabular}{llllllllll}
\hline Figures & \multicolumn{10}{c}{$I_{11}, I_{12}, I_{13}, \ldots, I_{1 m}$} \\
\multirow{2}{*}{ Figure 18 } & 0.1871 & 0.7106 & 0.1567 & 0.6932 & 0.4379 & 0.4480 & 1.0000 & 0.4338 & 0.4231 \\
& 1.0000 & 0.4892 & 0.7669 & 0.4860 & 0.2315 & 0.4899 & 0.7194 & 0.4938 & 0.2399 \\
\hline \multirow{2}{*}{ Figure 19 } & 0.1459 & 0.7218 & 0.1877 & 0.7146 & 0.4559 & 0.4731 & 1.0000 & 0.4711 & 0.4810 \\
& 0.9929 & 0.5301 & 0.6492 & 0.5558 & 0.2240 & 0.5390 & 0.7055 & 0.5268 & 0.2150 \\
\hline \multirow{2}{*}{ Figure 20 } & 0.2448 & 0.7762 & 0.2366 & 0.7750 & 0.5061 & 0.5109 & 0.9978 & 0.5004 & 0.4976 \\
& 1.0000 & 0.5393 & 0.7340 & 0.5418 & 0.2109 & 0.5443 & 0.7325 & 0.5426 & 0.2175 \\
\hline \multirow{2}{*}{ Figure 21 } & 0.9247 & 0.9169 & 0.9288 & 1.0000 & 0.6720 & 0.8673 & 0.4502 & 0.5717 & 0.8955 \\
& 0.3602 & 0.3792 & 0.4759 & 0.4940 & 0.1510 & 0.5172 & 0.4845 & 0.5198 & $0.2038 ; I_{c}=0.6767$ \\
\hline
\end{tabular}

\section{Conflict of Interests}

The authors declare that there is no conflict of interests regarding the publication of this paper.

\section{References}

[1] D. Mandal, S. P. Ghoshal, and A. K. Bhattacharjee, "Design of concentric circular antenna array with central element feeding using Particle Swarm Optimization with Constriction Factor and Inertia Weight Approach and Evolutionary Programing technique," Journal of Infrared, Millimeter, and Terahertz Waves, vol. 31, no. 6, pp. 667-680, 2010.

[2] D. Mandal, S. P. Ghoshal, and A. K. Bhattacharjee, "Application of evolutionary optimization techniques for finding the optimal set of concentric circular antenna array," Expert Systems with Applications, vol. 38, no. 4, pp. 2942-2950, 2011.

[3] D. Mandal, B. Goswami, R. Kar, S. P. Ghoshal, and A. K. Bhattacharjee, "Application of bio-inspired optimization technique for finding the optimal set of concentric circular antenna array with central element feeding," ACEEE International Journal on Information Technology, vol. 2, no. 2, pp. 6-10, 2012.

[4] R. Das, "Concentric ring array," IEEE Transactions on Antennas and Propagation, vol. 14, no. 3, pp. 398-400, 1966.

[5] N. Goto and D. K. Cheng, "On the synthesis of concentric-ring arrays," Proceedings of the IEEE, vol. 58, no. 5, pp. 839-840, 1970.
[6] D. Huebner, "Design and optimization of small concentric ring arrays," in Proceedings of the IEEE Antennas and Propagation Society International Symposium, pp. 455-458, March 1978.

[7] G. Holtrup, A. Marguinaud, and J. Citerne, "Synthesis of electronically steerable antenna arrays with elements on concentric rings with reduced sidelobes," in Proceedings of the IEEE Antennas and Propagation Society International Symposium, vol. 3, pp. 800-803, Boston, Mass, USA, July 2001.

[8] M. I. Dessouky, H. A. Sharshar, and Y. A. Albagory, "Efficient sidelobe reduction technique for small-sized concentric circular arrays," Progress in Electromagnetics Research, vol. 65, pp. 187200, 2006.

[9] R. L. Haupt, "Optimized element spacing for low sidelobe concentric ring arrays," IEEE Transactions on Antennas and Propagation, vol. 56, no. 1, pp. 266-268, 2008.

[10] D. Mandal, S. P. Ghoshal, and A. K. Bhattacharjee, "Improved swarm intelligence based optimal design of concentric circular antenna array," in Proceedings of the Applied Electromagnetics Conference (AEMC '09), pp. 1-4, Kolkata, India, December 2009.

[11] D. Mandal, S. P. Ghoshal, and A. K. Bhattacharjee, "Swarm intelligence based optimal design of concentric circular antenna array," Journal of Electrical Engineering, vol. 10, no. 3, pp. 30-39, 2010.

[12] D. Mandal, S. P. Ghoshal, and A. K. Bhattacharjee, "Radiation pattern optimization for concentric circular antenna array with 
central element feeding using craziness-based particle swarm optimization," International Journal of RF and Microwave Computer-Aided Engineering, vol. 20, no. 5, pp. 577-586, 2010.

[13] D. Mandal, A. Majumdar, R. Kar, and S. P. Ghoshal, “Thinned concentric circular array antennas synthesis using genetic algorithm," in Proceedings of the IEEE Student Conference on Research and Development (SCOReD '11), pp. 194-198, Cyberjaya, Malaysia, December 2011.

[14] E. I. Elsaidy, M. I. Dessouky, S. Khamis, and Y. A. Albagory, "Concentric circular antenna array synthesis using comprehensive learning particle swarm optimizer," Progress in Electromagnetics Research Letters, vol. 29, pp. 1-13, 2012.

[15] U. Singh and T. S. Kamal, "Synthesis of thinned planar concentric circular antenna arrays using biogeography-based optimisation," IET Microwaves, Antennas and Propagation, vol. 6, no. 7, pp. 822-829, 2012.

[16] U. Singh and T. S. Kamal, "Concentric circular antenna array synthesis using biogeography based optimization," Majlesi Journal of Electrical Engineering, vol. 6, no. 1, pp. 48-55, 2012.

[17] S. Biswas, D. Bose, S. Das, and S. Kundu, "Decompositionbased evolutionary multi-objective optimization approach to the design of concentric circular antenna arrays," Progress in Electromagnetics Research B, no. 52, pp. 185-205, 2013.

[18] H. Guo, C.-J. Guo, Y. Qu, and J. Ding, "Pattern synthesis of concentric circular antenna array by nonlinear least-square method," Progress In Electromagnetics Research B, vol. 50, pp. 331-346, 2013.

[19] R. L. Haupt, "Phase-only adaptive nulling with a genetic algorithm," IEEE Transactions on Antennas and Propagation, vol. 45, no. 6, pp. 1009-1015, 1997.

[20] W. Liao and F. Chu, "Array pattern nulling by phase and position perturbations with the use of the genetic algorithm," Microwave and Optical Technology Letters, vol. 15, no. 4, pp. 251-256, 1997.

[21] D. Karaboga, K. Guney, and A. Akdagli, "Antenna array pattern nulling by controlling both amplitude and phase using modified touring ant colony optimization algorithm," International Journal of Electronics, vol. 91, no. 4, pp. 241-251, 2004.

[22] A. A. Akdagli, K. Guney, and D. Karaboga, "Touring ant colony optimization algorithm for shaped-beam pattern synthesis of linear antenna," Electromagnetics, vol. 26, no. 8, pp. 615-628, 2006.

[23] N. B. Jin and Y. Rahmat-Samii, "Advances in particle swarm optimization for antenna designs: real-number, binary, singleobjective and multiobjective implementations," IEEE Transactions on Antennas and Propagation, vol. 55, no. 3, pp. 556-567, 2007.

[24] M. M. Khodier and C. G. Christodoulou, "Linear array geometry synthesis with minimum sidelobe level and null control using particle swarm optimization," IEEE Transactions on Antennas and Propagation, vol. 53, no. 8, pp. 2674-2679, 2005.

[25] S. W. Yang, Y. B. Gan, and A. Y. Qing, "Antenna-array pattern nulling using a differential evolution algorithm," International Journal of RF and Microwave Computer-Aided Engineering, vol. 14, no. 1, pp. 57-63, 2004.

[26] K. Guney and S. Basbug, "Null synthesis of time-modulated circular antenna arrays using an improved differential evolution algorithm," IEEE Antennas and Wireless Propagation Letters, vol. 12, pp. 817-820, 2013.

[27] A. Akdagli and K. Guney, "Shaped-beam pattern synthesis of equally and unequally spaced linear antenna arrays using a modified tabu search algorithm," Microwave and Optical Technology Letters, vol. 36, no. 1, pp. 16-20, 2003.
[28] A. Akdagli, K. Guney, and B. Babayigit, "Clonal selection algorithm for design of reconfigurable antenna array with discrete phase shifters," Journal of Electromagnetic Waves and Applications, vol. 21, no. 2, pp. 215-227, 2007.

[29] K. Guney and M. Onay, "Bees algorithm for interference suppression of linear antenna arrays by controlling the positions of selected elements," Journal of Communications Technology and Electronics, vol. 58, no. 12, pp. 1147-1156, 2013.

[30] K. Guney and M. Onay, "Synthesis of thinned linear antenna arrays using bees algorithm," Microwave and Optical Technology Letters, vol. 53, no. 4, pp. 795-799, 2011.

[31] K. Guney and M. Onay, "Optimal synthesis of linear antenna arrays using a harmony search algorithm," Expert Systems with Applications, vol. 38, no. 12, pp. 15455-15462, 2011.

[32] K. Guney, A. Durmus, and S. Basbug, "A plant growth simulation algorithm for pattern nulling of linear antenna arrays by amplitude control," Progress In Electromagnetics Research B, no. 17, pp. 69-84, 2009.

[33] K. Guney and S. Basbug, "Seeker optimization algorithm for interference suppression of linear antenna arrays by controlling position-only, phase-only, and amplitude-only," International Journal of RF and Microwave Computer-Aided Engineering, vol. 21, no. 5, pp. 505-518, 2011.

[34] K. Guney and S. Basbug, "Bacterial foraging algorithm for null synthesizing of linear antenna arrays by controlling only the element positions," Journal of Optimization Theory and Applications, vol. 150, no. 3, pp. 635-656, 2011.

[35] P. Civicioglu, "Backtracking search optimization algorithm for numerical optimization problems," Applied Mathematics and Computation, vol. 219, no. 15, pp. 8121-8144, 2013.

[36] P. Civicioglu, "Transforming geocentric cartesian coordinates to geodetic coordinates by using differential search algorithm," Computers and Geosciences, vol. 46, pp. 229-247, 2012.

[37] K. M. Passino, "Biomimicry of bacterial foraging for distributed optimization and control," IEEE Control Systems Magazine, vol. 22, no. 3, pp. 52-67, 2002.

[38] P. Civicioglu, "Circular antenna array design by using evolutionary search algorithms," Progress in Electromagnetics Research B, vol. 54, pp. 265-284, 2013. 

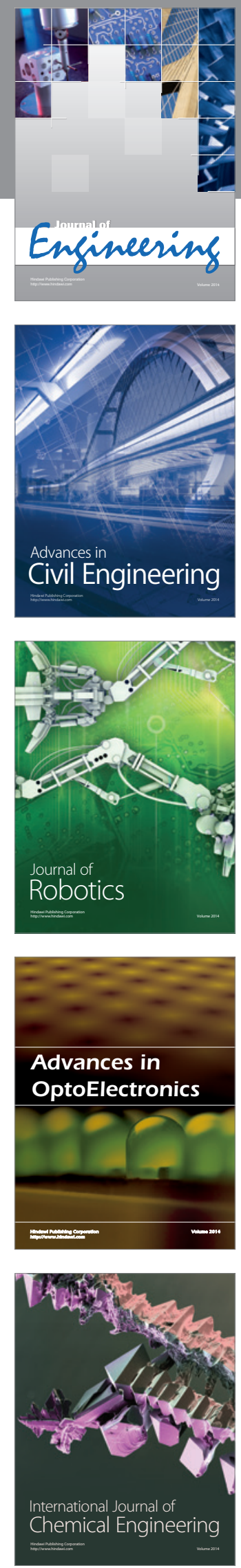

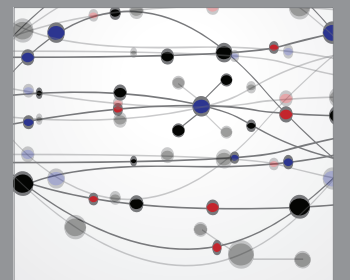

The Scientific World Journal
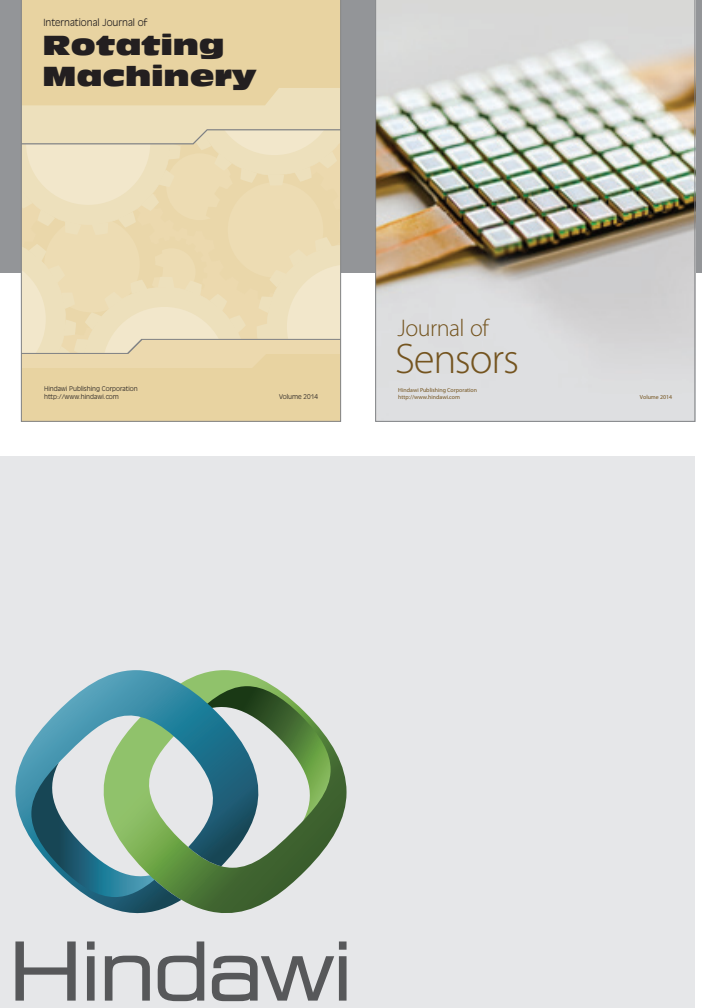

Submit your manuscripts at http://www.hindawi.com
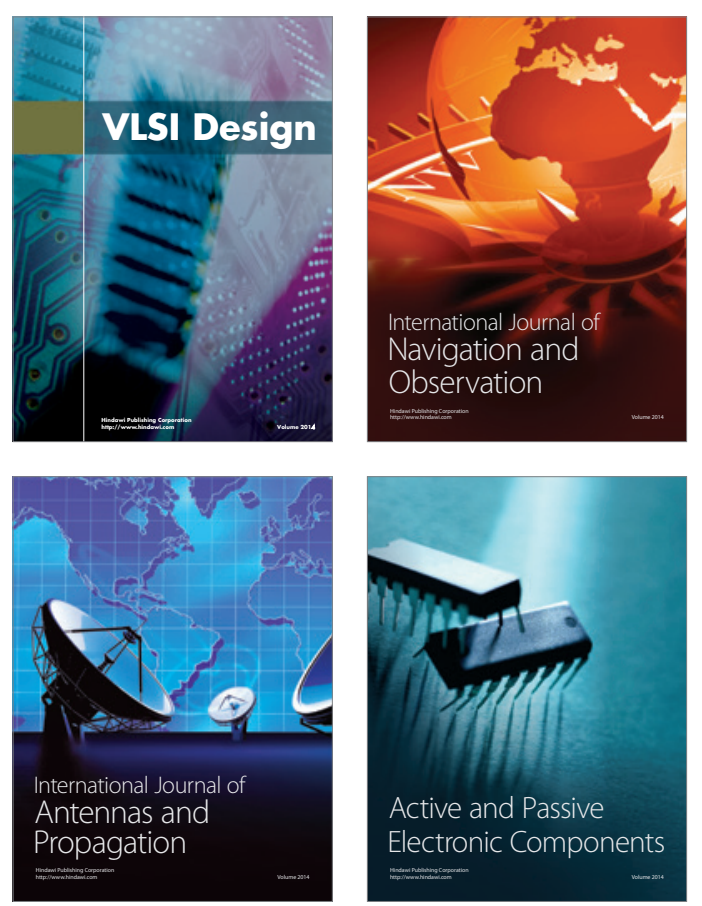
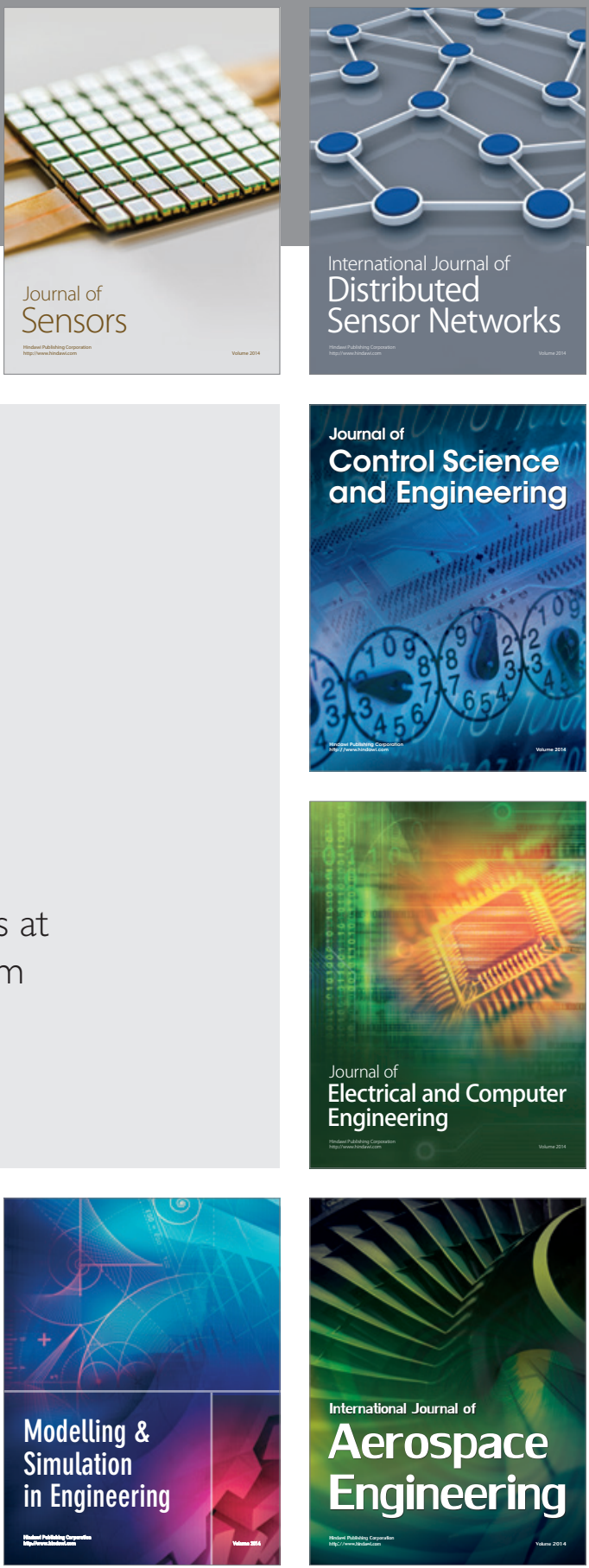

Journal of

Control Science

and Engineering
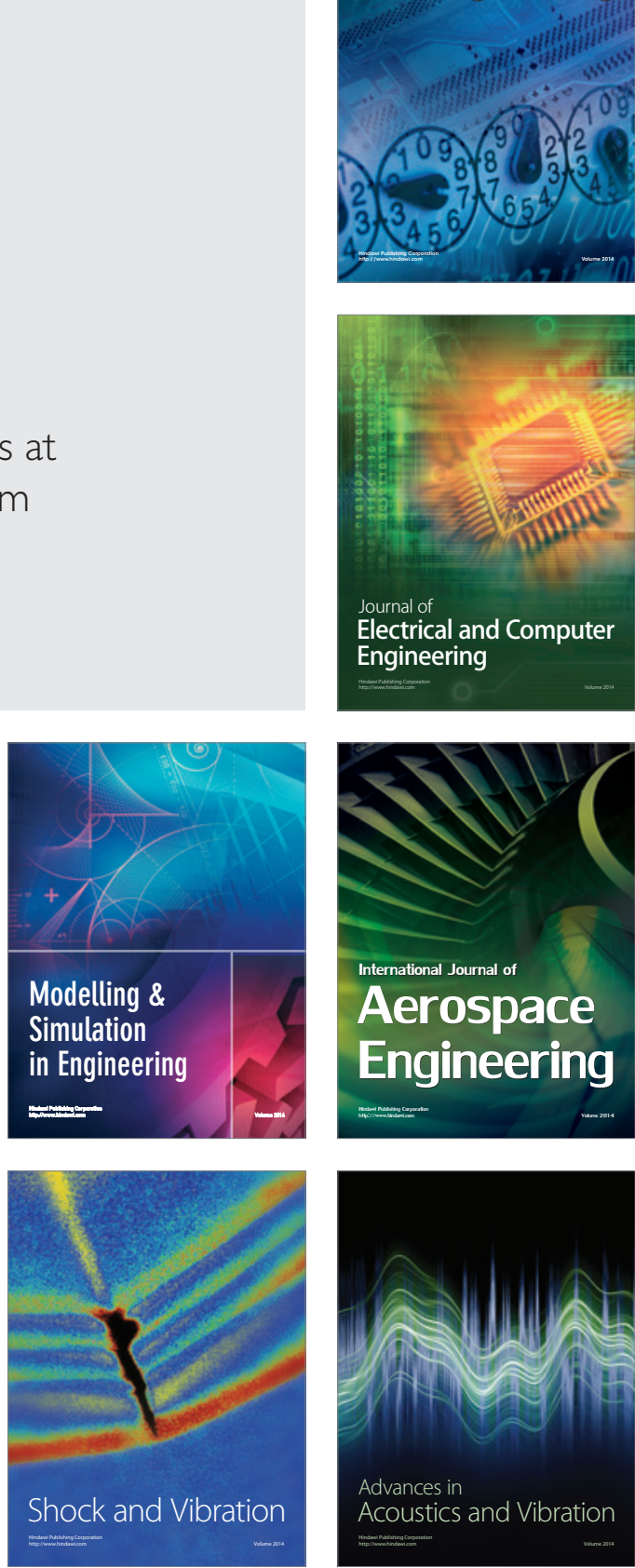